\title{
Have Sham Trials Put Current Cardiovascular Guidelines to Shame?
}

\section{Commentary}

The recently reported ORBITA study created a stir in the cardiovascular world suggesting that there was no indication for coronary stenting in the treatment of patients with stable angina [1]. This double blinded, randomized trial demonstrated that percutaneous coronary intervention did not increase exercise time compared to optimal medical treatment. In an accompanying editorial, a recommendation was made for downgrading the guideline recommendations for PCI in stable angina suggesting that this was the "last nail in the coffin for PCI in stable angina" [2]. As important as the findings of this study, was the methodology by which the study was conducted. In this study, 200 patients with stable Class II or III angina and severe coronary stenosis in a single vessel underwent six weeks of medical optimization. After medical treatment, patients were randomly assigned to a drug-eluting stent placement or a sham interventional procedure. The primary outcome was a change in exercise time on a treadmill at six weeks. Secondary endpoints included a change in exercise to $1 \mathrm{~mm}$ ST-segment depression, angina severity as assessed by Canadian Cardiovascular Society class, physical limitation, angina stability and frequency, Duke Treadmill score and dobutamine stress echocardiography (DSE) wall motion score index. Other than a significant improvement in DSE wall motion score, this was a negative study with respect to the primary and all other secondary endpoints. ORBITA was the first sham study in PCI and lauded in this approach to avoid the potential placebo effect of procedures such as PCI. Some have gone so far to declare that most future studies in coronary interventions should include sham procedures.

ORBITA was not the first sham control study to be performed in cardiovascular disease or for that matter in the treatment of angina pectoris. In 1959, internal mammary artery ligature versus a sham operation revealed no difference symptoms for treatment of angina pectoris [3]. It was hypothesized that mammary ligation increased coronary flow by the development of collaterals thus relieving angina. In this small study of 17 patients with severe angina, bilateral skin incision was as effective in reducing angina compared to bilateral mammary ligation. In addition, there was no change in exercise time to angina or the use of sublingual nitroglycerin between the two groups. These findings suggested that any benefit from mammary ligations more likely to be on a psychological basis and not a physiologic basis. The placebo effect was further tested in treatment of angina pectoris using percutaneous laser myocardial revascularization. Surgical transmyocardial laser revascularization was developed to address a population of patients with severe angina not amenable to percutaneous coronary intervention or coronary bypass surgery. Surgical trials had demonstrated improved angina and exercise tolerance and thus a less invasive catheter based laser revascularization approach was developed. In a randomized trial of 298 patients, treatment with percutaneous myocardial laser revascularization provided
David L. Fischman* and Michael P. Savage

Cardiac Catheterization Laboratory, Thomas Jefferson University Hospitals, Philadelphia, USA

*Author for correspondence: Tel.: +1(215) 955-5050 David.Fischman@jefferson.edu Submitted: March 06, 2018 Accepted: March 21, 2018

Published online: March 28, 2018 
no benefit compared to a similar sham procedure in patient's blinded to their assigned treatment [4]. There was no difference in the primary endpoint of exercise duration nor in the secondary endpoints of exercise time to the onset of chest pain, exercise time to the appearance of ST-segment changes at 6 and 12 months or frequency of angina symptoms.

In addition to placebo controlled trials assessing treatment of angina pectoris, interventional cardiologists have been in the forefront other sham-controlled trials for non-coronary interventions. In the PREMIUM trial, PFO closure revealed no reduction in the frequency of migraine headaches compared to sham procedure in a double-blind randomized trial of 230 patients [5]. The sham portion of this study was a right heart catheterization. In the Simplicity III trial patients with resistant hypertension were randomized to renal denervation or sham procedure [6]. In this study of 535 patients, there was no difference in the primary endpoint of a reduction in office or ambulatory systolic blood pressure at 6 months. The findings of this placebo controlled randomized trial contradicted previous trials conducted without sham controls which had demonstrated a significant improvement in blood pressure.

The findings of above mentioned studies underscore the importance of the utilization of sham controls in the assessment of procedures that are invasive in nature. These studies highlight the perceived effect that an intervention may have on one's subjective feelings. Having undergone an invasive procedure which entailed risks of complications, patients may be especially susceptible to a placebo effect. A systematic review of 53 placebo controlled trials of surgical procedures found that in over half the surgical intervention was not more effective that a sham procedure [7]. In ORBITA the thought that one received the intended treatment resulted in symptomatic improvement comparable to that of those who actually received the treatment. That ORBITA and similar studies should lead to the conclusion that most interventional devices

\section{References}

1. Al-Lamee R, Thompson D, Dehbi HM, et al. Percutaneous coronary intervention in stable angina (ORBITA): a doubleblind, randomized controlled trial. Lancet. 391: 31-40 (2018).

2. Brown D, Redberg RF. Last nail in the coffin for PCI in stable angina? Lancet. 391: 3-4 (2018).

3. Cobb LA, Thomas GI, Dillard DH, et al. An evaluation of should undergo the rigors of a sham control study to eliminate the placebo effect may be an overstatement. It is important to recognize that sham procedures are not without risk to the patient and could infringe on the concept of do no harm. Placing a catheter into the coronary vascularization imposes a risk of death, myocardial infarction (MI), stroke or bleeding to name some of the most serious complications. Thus, we must be cautious to balance these risks against the advantages of the sham procedure when incorporating such a methodology the endpoint to be achieved should be clearly worth the risk.

There is no argument that the perceived notion that one may have received a therapy lends to the general sense of well-being. Conversely, patients in a randomized interventional trial who are not blinded and therefore aware that they did not receive the active treatment may be more prone to experience recurrent symptoms. This underpins the criticism of the FAME2 trial which compared FFR guided PCI to medical therapy alone in patients with stable CAD [8]. The composite endpoint was significantly more frequent in the medical therapy group but this finding was driven by repeat revascularization procedures due to recurrent symptoms; in contrast, there was no significant difference between the two groups in death or MI. In many studies in coronary heart disease subjective feelings should not be the primary endpoint, but rather should recognize the hard endpoints that include death and myocardial infarction if we are truly to balance the benefit/risk ratio. To date no trial has demonstrated a reduction in death or MI in elective coronary stenting; however, these trials have had major limitations including being underpowered to detect mortality differences and high cross-over rates from medical therapy to PCI. Clearly ORBITA demonstrated a lack a benefit at six weeks but longer term follow-up data in a trial sufficiently large enough to determine death and MI outcomes is necessary prior to declaring the demise of stenting in stable angina and a need for a radical change in the guidelines.

internal mammary ligation by a double blind technic. $N$ Engl J Med. 260: 1115 (1959)

4. Leon, MD, Kornowski R, Downey WE, et al. A Blinded, Randomized Trial of Percutaneous Laser Myocardial Revascularization in Patients with Severe Coronary Disease. J Am Coll Cardiol. 46: 1812-1819 (2005)

5. Tobis JM, Charles A, Silberstein SD, et al. Percutaneous Closure of Patent Foramen Ovale in Patients with Migraine: 
The PREMIUM Trial. J Am Coll Cardiol. 70: 2766-2774 (2017)

6. Bhatt DL, Kandzari DE, O'Neill WW, et al. A Controlled Tria of Renal Denervation for Resistant Hypertension. N Engl J Med. 370: 1393-1401 (2014).

7. Wartolowska K, Judge A, Hopewell S, et al. Use of placebo controls in the evaluation of surgery:systematic review. BMJ. 348: g3253 (2014).

8. De Bruyne B, Pijls NHJ, Kalesan B, et al. Fractional Flow Reserve-Guided PCI versus Medical Therapy in Stable Coronary Disease. N Engl J Med. 367: 991-1001 (2012). 